\title{
AN $L_{p}$-INEQUALITY FOR ANTICOMMUTATORS
}

\author{
ÉRIC RICARD
}

\begin{abstract}
We prove a basic inequality involving anticommutators in noncommutative $L_{p^{-}}$ spaces. We use it to complete our study of the noncommutative Mazur maps from $L_{p}$ to $L_{q}$ showing that they are Lipschitz on balls when $0<q<p<\infty$.
\end{abstract}

\section{INTRODUCTION}

This short note deals with noncommutative integration in von Neumann algebras. We refer to 9 for basic definitions and notation in the semifinite or type III setting. We will simply denote by $L_{p}$ the noncommutative $L_{p}$-space associated to a semifinite von Neumann algebra $\mathcal{M}$ with a trace $\tau$ or a general von Neumann algebra $\mathcal{M}$ with a weight $\varphi$.

The Mazur maps $M_{p, q}$ are bijections between $L_{p}$ and $L_{q}$ defined by $M_{p, q}(f)=f|f|^{p / q-1}$ for $0<p, q<\infty$. They are known to be uniformly continuous on balls by [10. Very few precise estimates were known. Kosaki gave some of them [7, 6] and the work of Alexandrov and Peller [1] on the functional calculus can be used for the case $1<q<p$ on Schatten classes. In several papers, we tried to quantify this continuity. In [11, we treated the Banach space case $1 \leqslant p, q<\infty$. Just as in the commutative case, when $p>q, M_{p, q}$ is globally $\frac{p}{q}$-Hölder and simply Lipschitz on balls when $q<p$. The involved tools make a crucial use of convexity that is not available below exponent 1 . For instance, unital completely positive Schur multipliers are no longer bounded on the Schatten $p$-class $S_{p}$ when $p<1$. They are used in lot of inequalities in noncommutative $L_{p}$ and are closely related to functional calculus; this explains why the quasi-Banach situation is much more difficult to handle and pretty much unexplored. The main inequality of 8 used plurisubharmonicity of the $L_{p}$-norm (in the finite case) to overcome this lack of convexity. It leads to non optimal estimates for the modulus of continuity of $M_{p, q}$ is the quasi-Banach case. To deal with $p<q$ in [12, we used precise estimates on the boundedness of Schur multipliers on $S_{p}$ as well as a suitable decomposition of operators to get the optimal rate. The main motivation for this paper is to settle the remaining case $q<p$ when $q<1$.

Our main argument is a variation on [8] combined with general techniques from [11. Our main inequality is written in terms of an anticommutator estimate which barely says that a kind of weighted triangular truncation is bounded from $L_{p}$ to $L_{q}$. Not so surprisingly, it implies the $L_{p}-L_{q}$ boundedness of some Schur type multipliers that turn out to be enough to deal with Mazur maps. The proofs of the inequalities are made for semifinite von Neumann algebras (finite is enough by [11). The general case can be obtained using the Haagerup reduction principle as in [11.

We will focus only on the relevant inequalities and skip technical approximation arguments that can be found in details in $\left[8,11,12\right.$. As often, we write $C_{p, q}$ for constants that depends on the parameter $p, q$ and may differ from line to line.

\section{INEQUALITIES}

2.1. Main result. Let $(\mathcal{M}, \tau)$ be a semifinite von Neumann algebra.

Theorem 2.1. Fix $\alpha>0,0<s<\infty$ and $0<r \leqslant \infty$. Let $p$ be so that $\frac{1}{p}=\frac{1}{s}+\frac{1}{r}$ and $q$ so that $\frac{1}{q}=\frac{1+\alpha}{s}+\frac{1}{r}$. Then there is a constant $C_{\alpha, q}$ so that for any $d \in L_{s}^{+}$and $x \in L_{r}$ :

$$
\left\|x d^{1+\alpha}\right\|_{q} \leqslant C_{\alpha, q}\|d\|_{s}^{\alpha} \cdot\|d x+x d\|_{p} .
$$

2010 Mathematics Subject Classification: 46L51; 47B10.

Key words: Noncommutative $L_{p}$-spaces, functional calculus. 
The proof will be a variation on the arguments of [8]. We will need a bit of notation. Let $\Delta$ be the unit strip in $\mathbb{C}$ with boundaries $\partial_{k}$ for $k=0,1$ :

$$
\Delta=\{z \in \mathbb{C} ; 0<\operatorname{Re} z<1\} ; \quad \partial_{i}=\{z \in \mathbb{C} ; \operatorname{Re} z=k\} .
$$

For $0<\gamma<1$, we will denote by $\mathbb{P}^{\gamma}$ the Poisson measure on the boundary of $\Delta$ relative to the point $\gamma$. It is explicitly given by densities on $\partial_{k}$ with respect to the Lebesgue measure, for $t \in \mathbb{R}$ :

$$
Q_{\gamma}(k+i t)=\frac{\sin (\gamma \pi)}{2\left(\cosh (\pi t)-(-1)^{k} \cos (\gamma \pi)\right)} .
$$

For a $A \subset \partial_{0} \cup \partial_{1}$, we write $2 . A$ for its dilation by a factor 2 in the imaginary direction.

Lemma 2.2. There is a constant $C_{\gamma}>0$, such that for any borel set $A \subset \partial_{0} \cup \partial_{1}$, one has $\mathbb{P}^{\gamma}(2 . A) \leqslant C_{\gamma} \mathbb{P}^{\gamma}(A)$.

Proof. We need the obvious fact that $\mathbb{P}^{\gamma}$ is equivalent to $\mu$ the measure on $\partial_{0} \cup \partial_{1}$ with density $Q(k+i t)=\frac{1}{\cosh (\pi t)}$ with a constant that depends only on $\gamma$ :

$k=0,1, \quad \forall t \in \mathbb{R}, \quad Q_{\gamma}(k+i t) \leqslant \frac{\sin (\gamma \pi)}{2(1-|\cos (\gamma \pi)|)} Q(k+i t), \quad Q(k+i t) \leqslant \frac{4}{\sin (\gamma \pi)} Q_{\gamma}(k+i t)$.

Thus it suffices to prove the lemma for $\mu$. But for any borel set $B$ in $\mathbb{R}$

$$
\int_{2 B} \frac{d t}{\cosh (\pi t)}=2 \int_{B} \frac{d u}{\cosh (2 \pi u)} \leqslant 2 \int_{B} \frac{d t}{\cosh (\pi t)} .
$$

It follows that $\mu(2 . A) \leqslant 2 \mu(A)$ for any borel set of $\partial_{0} \cup \partial_{1}$.

Let us recall the complex uniform convexity of $L_{q}$ from [14, see also [8, Remark 2.8]. To avoid technical discussions, we will consider only the set $A F(\Delta)$ of holomorphic functions $F: \Delta \rightarrow \mathcal{M}$ that are bounded and extend continuously on $\partial_{k}, k=0,1$ with values in a given finite von Neumann subalgebra of $\mathcal{M}$ (depending on $F$ ). For such an $F$ and $0<\gamma<1$, we denote by $\|F\|_{L_{q}^{\gamma}}$ its $L_{q}$ norm in with respect to the measure $\mathbb{P}^{\gamma}$ considering it with values in $L_{q}(\mathcal{M}, \tau)$.

Theorem 2.3. Let $0<q \leqslant 2$, there is a constant $\delta_{q}>0$ such that for any $F \in A F(\mathcal{M})$ and $0<\gamma<1$ :

$$
\|F(\gamma)\|_{q}^{2}+\delta_{q}\|F-F(\gamma)\|_{L_{q}^{\gamma}}^{2} \leqslant\|F\|_{L_{q}^{\gamma}}^{2}
$$

Proof of Theorem [2.1. By standard approximation arguments (see [8, Proof of 2.1]), we can assume that $d$ and $x$ sit in a finite von Neumann algebra and that $d$ is invertible with $\|d\|_{s}=1$ and has a finite discrete spectrum. Moreover using a $2 \times 2$ matrix trick, we can assume $x=x^{*}$ loosing on the constant $C_{\alpha, q}$. This is to ensure that $\left\|x d^{1+\alpha}\right\|_{q}=\left\|d^{1+\alpha} x\right\|_{q}$.

We start by the most difficult case $q \leqslant 1$.

Let $1>\eta>0$ to be fixed latter and set $\gamma=\frac{\alpha}{1+\alpha} \in(0,1)$. We first assume that $\|x d+d x\|_{p} \leqslant$ $\eta\left\|x d^{1+\alpha}\right\|_{q}$.

By the $q$-triangle and the Hölder inequalities, we have

$$
\left\|x d^{1+\alpha}\right\|_{q}^{q}=\left\|d^{1+\alpha} x\right\|_{q}^{q} \leqslant\left\|d^{\alpha} x d\right\|_{q}^{q}+\left\|d^{\alpha}(x d+d x)\right\|_{q}^{q} \leqslant\left\|d^{\alpha} x d\right\|_{q}^{q}+\eta^{q}\left\|x d^{1+\alpha}\right\|_{q}^{q} .
$$

Thus $\left(1-\eta^{q}\right)\left\|x d^{1+\alpha}\right\|_{q}^{q} \leqslant\left\|d^{\alpha} x d\right\|_{q}^{q}$.

Because of all the assumptions we made on $d$, the function given by $F(z)=d^{(1+\alpha) z} x d^{(1+\alpha)(1-z)}$ is in $A F(\mathcal{M})$. We have $F(\gamma)=d^{\alpha} x d$ and moreover for all $t \in \mathbb{R}$ as $x=x^{*}$

$$
\|F(i t)\|_{q}=\left\|x d^{1+\alpha}\right\|_{q}=\left\|d^{1+\alpha} x\right\|_{q}=\|F(1+i t)\|_{q}
$$

thus $\|F\|_{L_{q}^{\gamma}}=\left\|x d^{1+\alpha}\right\|_{q}$. By Theorem 2.3 .

$$
\delta_{q}\left\|F-d^{\alpha} x d\right\|_{L_{q}^{\gamma}}^{2} \leqslant\left\|x d^{1+\alpha}\right\|_{q}^{2}-\left\|d^{\alpha} x d\right\|_{q}^{2} \leqslant\left(1-\left(1-\eta^{q}\right)^{2 / q}\right)\left\|x d^{1+\alpha}\right\|_{q}^{2} .
$$

Let $\varepsilon=2.6^{-1 / q}$ and $A=\left\{z \in \partial_{0} \cup \partial_{1} \mid\left\|F(z)-d^{\alpha} x d\right\|_{q} \geqslant \varepsilon\left\|x d^{1+\alpha}\right\|_{q}\right\}$.

By the Markov inequality,

$$
\mathbb{P}^{\gamma}(A) \leqslant \frac{\left(1-\left(1-\eta^{q}\right)^{2 / q}\right)^{q / 2}}{\delta_{q}^{q / 2} \varepsilon^{q}} .
$$


By Lemma 2.2, if $\eta$ is chosen small enough, we have that $\mathbb{P}^{\gamma}(A \cup 2 . A) \leqslant \gamma / 2$. Since $\mathbb{P}^{\gamma}\left(\delta_{1}\right)=\gamma$, we can find $z \in \delta_{1} \backslash(A \cup 2 A)$. Let $z=1+2 i t$ with $t \in \mathbb{R}$ and put $u_{h}=d^{(1+\alpha) i h}$ which is obviously an unitary commuting with $d$ for all $h \in \mathbb{R}$. By the definition of $2 . A$ and $A$ we have

$$
\left\|d^{1+\alpha} u_{t} x u_{-t}-d^{\alpha} x d\right\|_{q},\left\|d^{1+\alpha} u_{2 t} x u_{-2 t}-d^{\alpha} x d\right\|_{q} \leqslant \varepsilon\left\|x d^{1+\alpha}\right\|_{q} .
$$

By unitary invariance, the second inequality is $\left\|d^{1+\alpha} u_{t} x u_{-t}-u_{-t} d^{\alpha} x d u_{t}\right\|_{q} \leqslant \varepsilon\left\|x d^{1+\alpha}\right\|_{q}$, thus

$$
\left\|d^{\alpha} x d-u_{-t} d^{\alpha} x d u_{t}\right\|_{q}=\left\|d^{\alpha} x d-u_{t} d^{\alpha} x d u_{-t}\right\|_{q} \leqslant 2^{1 / q} \varepsilon\left\|x d^{1+\alpha}\right\|_{q} .
$$

Using the first inequality and unitary invariance again

$$
\left\|d^{1+\alpha} x-d^{\alpha} x d\right\|_{q} \leqslant 3^{1 / q} \varepsilon\left\|x d^{1+\alpha}\right\|_{q} .
$$

Then by the $q$-triangle and the Hölder inequalities

$$
\left\|2 d^{1+\alpha} x\right\|_{q}^{q} \leqslant\left\|d^{1+\alpha} x+d^{\alpha} x d\right\|_{q}^{q}+3 \varepsilon^{q}\left\|x d^{1+\alpha}\right\|_{q}^{q} \leqslant\|d x+x d\|_{p}^{q}+3 \varepsilon^{q}\left\|x d^{1+\alpha}\right\|_{q}^{q} .
$$

We finally get $\|d x+x d\|_{p} \geqslant\left(2^{q}-3 \varepsilon^{q}\right)^{1 / q}\left\|x d^{1+\alpha}\right\|_{q}=2^{1-1 / q}\left\|x d^{1+\alpha}\right\|_{q}$ assuming $\|x d+d x\|_{p} \leqslant$ $\eta\left\|x d^{1+\alpha}\right\|_{q}$.

Thus for $\eta$ chosen as above, we must have for all $x \in L_{r}$ and $d \in L_{s}^{+}$with $\|d\|_{s}=1$ :

$$
\|d x+x d\|_{p} \geqslant \min \left\{\eta, 2^{1-1 / q}\right\} \cdot\left\|x d^{1+\alpha}\right\|_{q}
$$

The case $1 \leqslant q \leqslant 2$ can be done in the exact same way replacing the $q$-triangle inequality by the usual triangle inequality.

Actually when $q>1$, then $p>1$ and we have a stronger inequality

$$
\|x d\|_{p} \leqslant C_{p}\|d x+x d\|_{p}
$$

This follows from the complete boundedness of triangular projections on $L_{p}$, see [4]. Thus we get the inequality with constant $C_{p} \leqslant C_{(1+\alpha) q}$. The constant can even be made independent of $\alpha$, we sketch an argument. As in Corollary 2.3 of [13, for any $n$ and any sequence $d_{i}>0$, the matrix $\left(\frac{d_{i}^{1+\alpha}+d_{j}^{1+\alpha}}{\left(d_{i}+d_{j}\right)\left(d_{i}^{\alpha}+d_{j}^{\alpha}\right)}\right)$ defines a Schur multiplier on $M_{n}$ with norm less than $\frac{5}{2}$. It follows

$$
\left\|x d^{1+\alpha}\right\|_{q} \leqslant C_{q}\left\|d^{1+\alpha} x+x d^{1+\alpha}\right\|_{q} \leqslant \frac{5}{2} C_{q}\left\|d^{1+\alpha} x+d^{\alpha} x d+d x d^{\alpha}+x d^{1+\alpha}\right\|_{q}
$$

Thus using the Hölder and the triangle inequality, we end up with

$$
\left\|x d^{1+\alpha}\right\|_{q} \leqslant 5 C_{q}\|d\|_{s}^{\alpha}\|d x+x d\|_{p}
$$

Remark 2.4. For integer values of $\alpha$, it is possible to give a simple algebraic proof using the same kind of ideas but without using the complex uniform convexity.

Let us give an easy corollary that improves an estimate from [8] which is relevant only for $p \leqslant 1$ :

Corollary 2.5. Let $0<\varepsilon<1,0<s<\infty$ and $0<r \leqslant \infty$ let $\frac{1}{p}=\frac{1}{s}+\frac{1}{r}$. Then there is a constant $C_{\varepsilon, p}$ so that for any $d \in L_{s}^{+}$and $x \in L_{r}$ :

$$
\|x d\|_{p} \leqslant C_{\varepsilon, p}\left(\|d\|_{s}\|x\|_{r}\right)^{\varepsilon} \cdot\|d x+x d\|_{p}^{1-\varepsilon} .
$$

Proof. This is a consequence of the complex interpolation [8, Lemma 2.5] for the function $F(z)=$ $x d^{z /(1-\varepsilon)}$ (with an approximation argument as above to make it in $A F(\mathcal{M})$ ). Set $q$ so that $\frac{1}{q}=$ $\frac{1-\varepsilon}{p}+\frac{\varepsilon}{r}$, we have that $\|x d\|_{p} \leqslant\left\|x d^{1 /(1-\varepsilon)}\right\|_{q}^{1-\varepsilon}\|x\|_{r}^{\varepsilon}$. We can conclude applying Theorem 2.1 with $\alpha=\frac{\varepsilon}{1-\varepsilon}$. 
2.2. Applications to the Mazur maps. On a semifinite von Neumann algebra $(\mathcal{M}, \tau)$, the left Mazur map $M_{p, q}: L_{p} \rightarrow L_{q}$ is given by $M_{p, q}(f)=f|f|^{p / q-1}$. It is a uniformly continuous bijection on balls by [10]. We determined its modulus of continuity in our previous papers [11, 12] except in the case $p>q$ when $q<1$. It still coincides with its commutative analogue:

Theorem 2.6. Let $(\mathcal{M}, \tau)$ be a semifinite von Neumann algebra, then for $q<p<\infty$, the Mazur map $M_{p, q}$ is Lipschitz on balls:

$$
\forall x, y \in L_{p}, \quad\left\|M_{p, q}(x)-M_{p, q}(y)\right\|_{q} \leqslant C_{p, q} \max \left\{\|x\|_{p},\|y\|_{p}\right\}^{p / q-1} \cdot\|x-y\|_{p} .
$$

From the arguments of the papers [11, 12, one needs to prove that

$$
\forall x \in \mathcal{M}, \forall d \in L_{p}^{+}, \quad\left\|x d^{p / q} \pm d^{p / q} x\right\|_{q} \leqslant C_{p, q}\|x d \pm d x\|_{p}\|d\|_{p}^{p / q-1} .
$$

The inequality (11) with the plus signs is an easy consequence of Theorem 2.1. The inequality with the minus sign is also equivalent to (see [11, Lemma 2.4])

$$
\forall x, y \in L_{p}^{+}, \quad\left\|x^{p / q}-y^{p / q}\right\|_{q} \leqslant C_{p, q}^{\prime} \max \left\{\|x\|_{p},\|y\|_{p}\right\}^{p / q-1} \cdot\|x-y\|_{p} .
$$

To prove (2), one can assume again that both $x$ and $y$ sit in a finite von Neumann algebra and are invertible with finite spectra. We will need an intermediate result.

We assume that $(\mathcal{M}, \tau)$ is finite and let $d=\sum_{i=1}^{n} d_{i} p_{i}$ with $d_{i}>0$ and where $p_{i}$ are orthogonal projections summing up to 1 . We define a map $T_{\beta, \gamma}^{d}$ on $\mathcal{M}$ for $0<\gamma<1$ and $\beta \geqslant 0$ by

$$
T_{\beta, \gamma}^{d}(\delta)=\sum_{i, j=1}^{n} \frac{d_{i}^{\gamma}-d_{j}^{\gamma}}{d_{i}-d_{j}} d_{i}^{\beta} d_{j}^{\beta} p_{i} \delta p_{j},
$$

when $i=j, \frac{d_{i}^{\gamma}-d_{j}^{\gamma}}{d_{i}-d_{j}}$ means $\gamma d_{i}^{\gamma-1}$. Note that $T_{\beta, \gamma}^{d}$ is completely positive when $0<\gamma<1$ as the matrix $\left(\frac{d_{i}^{\gamma}-d_{j}^{\gamma}}{d_{i}-d_{j}}\right)_{i, j=1}^{n}$ is positive when $0<\gamma \leqslant 1$.

We write $\alpha=2 \beta+\gamma-1$, and as in Theorem main with $0<s<\infty$ and $0<r \leqslant \infty$, we set $p$ so that $\frac{1}{p}=\frac{1}{s}+\frac{1}{r}$ and $q$ so that $\frac{1}{q}=\frac{1+\alpha}{s}+\frac{1}{r}$.

Lemma 2.7. With the above notation, for $1 / 2 \leqslant \gamma<1$ and $\beta>0$ so that $\alpha>0$ and $0<q \leqslant 1$, there is a constant $C_{\beta, \gamma}$ so that for any $\delta$ in $\mathcal{M}$

$$
\left\|T_{\beta, \gamma}^{d}(\delta)\right\|_{q} \leqslant C_{\beta, \gamma, q}\|\delta\|_{p}\|d\|_{s}^{\alpha} .
$$

Proof. We start with the case $\gamma=\frac{1}{2}$. In this case $\frac{d_{i}^{\gamma}-d_{j}^{\gamma}}{d_{i}-d_{j}}=\frac{1}{\sqrt{d_{i}}+\sqrt{d_{j}}}$. Since we assume $d$ invertible, we can make a change of variable by letting $\delta=\sqrt{d} x+x \sqrt{d}$. Then we want to prove that

$$
\left\|d^{\beta} x d^{\beta}\right\|_{q}=\left\|\sum_{i, j=1}^{n} d_{i}^{\beta} d_{j}^{\beta} p_{i} x p_{j}\right\|_{q} \leqslant C_{\beta, \gamma, q}\|\sqrt{d} x+x \sqrt{d}\|_{p} \cdot\|d\|_{s}^{\alpha} .
$$

Recall that $\|\cdot\|_{q}$ is plurisubharmonic (see [5, 14]) or simply by the Hölder inequality $\left\|d^{\beta} x d^{\beta}\right\|_{q} \leqslant$ $\max \left\{\left\|d^{2 \beta} x\right\|_{q},\left\|x d^{2 \beta}\right\|_{q}\right\}$. Note that $2 \beta>\frac{1}{2}$, we can write $4 \beta=1+2 \alpha$. We can apply Theorem 2.1 for $x$ and $x^{*}$ with $\sqrt{d}$ instead of $d, 2 \alpha$ instead of $\alpha$ and $2 s$ instead of $s$ to get

$$
\max \left\{\left\|x \sqrt{d}^{1+2 \alpha}\right\|_{q},\left\|\sqrt{d}^{1+2 \alpha} x\right\|_{q}\right\} \leqslant C_{2 \alpha, q}\|\sqrt{d}\|_{2 s}^{2 \alpha} \cdot\|\sqrt{d} x+x \sqrt{d}\|_{p}
$$

as $\frac{1}{q}=\frac{1}{p}+\frac{2 \alpha}{2 s}$. This is exactly the result.

Let $\frac{1}{2}<\gamma<1$. As we can decompose $\delta$ into the sum of 4 positive elements (with a control on the norm), it suffices to prove the inequality when $\delta \geqslant 0$. Put $v=1 /(2 \gamma)<1$, we use the relation

$$
T_{(1-v) / 2, v}^{d^{\gamma}} T_{\beta, \gamma}^{d}=T_{\beta+\gamma(1-v) / 2,1 / 2}^{d}=T_{\beta+(\gamma-1) / 2,1 / 2}^{d}
$$

The map $T_{(1-v) / 2, v}^{d^{\gamma}}$ is completely positive with $T_{(1-v) / 2, v}^{d^{\gamma}}(1)=v$. The Hansen-Pedersen inequality ensures that $S\left(y^{q}\right) \leqslant S(y)^{q}$ for $y \geqslant 0,0<q<1$, and $S$ unital completely positive. We apply it for $y=v T_{\beta, \gamma}^{d}(\delta)$ which is positive and $S=\frac{1}{v} T_{(1-v) / 2, v}^{d^{\gamma}}$ which is also trace preserving to get

$$
\left\|T_{\beta, \gamma}^{d}(\delta)\right\|_{q} \leqslant v^{1-q}\left\|T_{\beta+(\gamma-1) / 2,1 / 2}^{d}(\delta)\right\|_{q} .
$$

We apply the result for $\gamma=1 / 2$, because $\alpha=2 \beta+\gamma-1>0$ :

$$
\left\|T_{\beta, \gamma}^{d}(\delta)\right\|_{q} \leqslant C_{\beta, \gamma, q}\|\delta\|_{p}\|d\|_{s}^{\alpha} .
$$


Remark 2.8. One can get the lemma for $0<\gamma<1$ using compositions but we won't need it.

Remark 2.9. For $1 \leqslant \gamma \leqslant 2$, the function $x \mapsto x^{\gamma}$ is operator convex on $L_{p}^{+}$. If $x=y+\delta$ with $\delta \geqslant 0$ assuming $\|\delta\|_{p} \leqslant 1=\|x\|_{p}$, by convexity

$$
x^{\gamma}-y^{\gamma} \leqslant\left(1-\|\delta\|_{p}\right) y^{\gamma}+\|\delta\|_{p}\left(y+\frac{1}{\|\delta\|_{p}} \delta\right)^{\gamma}-y^{\gamma}
$$

Thus, there is an easy lipschitz norm control in $L_{p}$ for the positive part of $x^{\gamma}-y^{\gamma}$. To get another estimate for the negative part, one can use another operator inequality to reduce it to the derivative of the $\gamma$-power function at $y$ on $\delta$. This is the is the content of Lemma 2.7 which can be seen as a control of the defect of positivity of $x^{\gamma}-y^{\gamma}$.

End of proof of Theorem 2.6. We want to prove (2) when $(\mathcal{M}, \tau)$ is finite $x=\sum_{i=1}^{n} x_{i} p_{i}$ is positive invertible with a finite spectrum and similarly $y=\sum_{j=1}^{n} y_{j} q_{j}$.

We assume for the moment that $p<3 q / 2$ so that $p / q=1+t$ with $0<t<1 / 2$. We have

$$
\begin{aligned}
x^{p / q}-y^{p / q} & =\sum_{i=1}^{n} \sum_{j=1}^{m} \frac{x_{i}^{1+t}-y_{j}^{1+t}}{x_{i}-y_{j}} p_{i}(x-y) q_{j} \\
& =x^{t}(x-y)+(x-y) y^{t}-\sum_{i=1}^{n} \sum_{j=1}^{m} \frac{x_{i}^{1-t}-y_{j}^{1-t}}{x_{i}-y_{j}} x_{i}^{t} y_{j}^{t} p_{i}(x-y) q_{j}
\end{aligned}
$$

Let $z$ denote the last term of the sum. We use the standard $2 \times 2$ trick considering $M_{2}(\mathcal{M})$ with trace $\operatorname{Tr} \otimes \tau$. We let $d=\left[\begin{array}{ll}x & 0 \\ 0 & y\end{array}\right]$ and $\delta=\left[\begin{array}{cc}0 & x-y \\ 0 & 0\end{array}\right]$, by Lemma 2.7 with $\gamma=1-t, \beta=t$ $(\alpha=t>0)$ and $s=p$, we get

$$
\|z\|_{q}=\left\|T_{t, 1-t}^{d}(\delta)\right\|_{q} \leqslant C_{t, q}\|x-y\|_{p} \cdot\left(\|x\|_{p}^{p}+\|y\|_{p}^{p}\right)^{t / p} .
$$

To get the general case, it suffices to notice that $M_{q, r} M_{p, q}=M_{p, r}$ so that one can iterate the result when $0<t<1 / 2$ to get that for all $t>0$.

Compared with Lemma 2.7, it is even more direct to show, with the notation of the proof of Theorem 2.6. that the maps $\delta \mapsto \sum_{i=1}^{n} \sum_{j=1}^{m} \frac{x_{i}^{p / q}-y_{j}^{p / q}}{x_{i}+y_{j}} p_{i} \delta q_{j}$ is bounded from $L_{p}$ to $L_{q}$ when $p>q$. By the same arguments as in [12], one gets

Corollary 2.10. Let $(\mathcal{M}, \tau)$ be a semifinite von Neumann algebra, then for $q<p<\infty$ :

$$
\forall x, y \in L_{p}, \quad\left\||x|^{p / q}-|y|^{p / q}\right\|_{q} \leqslant C_{p, q} \max \left\{\|x\|_{p},\|y\|_{p}\right\}^{p / q-1} \cdot\|x-y\|_{p} .
$$

2.3. Extension to type III. Theorems 2.1 and 2.6 and their corollaries extend in the exact same form to type III von Neumann algebras using Haagerup's definition. This follows from the Haagerup reduction principle [3] (or 2] for weights). The arguments are given in the last section of 11 with the only difference that one has to avoid the use of conditional expectations but rather use simple approximations that are provided by the reduction principle $\left(\cup_{n} L_{p}\left(\mathcal{R}_{n}\right)\right.$ is dense in $\left.L_{p}(\mathcal{R})\right)$. The key point is that this reduction principle is compatible with the functional calculus and we know from [10] that powers and Mazur maps are continuous. We leave the details to the interested reader.

Acknowledgement. The author is supported by ANR-19-CE40-0002.

\section{REFERENCES}

[1] A. B. Aleksandrov and V. V. Peller. Functions of operators under perturbations of class $\mathbf{S}_{p}$. J. Funct. Anal., 258(11):3675-3724, 2010.

[2] Martijn Caspers, Javier Parcet, Mathilde Perrin, and Éric Ricard. Noncommutative de Leeuw theorems. Forum Math. Sigma, 3:e21, 59, 2015.

[3] Uffe Haagerup, Marius Junge, and Quanhua Xu. A reduction method for noncommutative $L_{p}$-spaces and applications. Trans. Amer. Math. Soc., 362(4):2125-2165, 2010.

[4] Marius Junge and Javier Parcet. Rosenthal's theorem for subspaces of noncommutative $L_{p}$. Duke Math. J., 141(1):75-122, 2008.

[5] N. J. Kalton. Plurisubharmonic functions on quasi-Banach spaces. Studia Math., 84(3):297-324, 1986. 
[6] Hideki Kosaki. Applications of uniform convexity of noncommutative $L^{p}$-spaces. Trans. Amer. Math. Soc., 283(1):265-282, 1984.

[7] Hideki Kosaki. On the continuity of the map $\varphi \rightarrow|\varphi|$ from the predual of a $W^{*}$-algebra. J. Funct. Anal., 59(1):123-131, 1984.

[8] Gilles Pisier and Éric Ricard. The non-commutative Khintchine inequalities for $0<p<1$. J. Inst. Math. Jussieu, 16(5):1103-1123, 2017.

[9] Gilles Pisier and Quanhua Xu. Non-commutative $L^{p}$-spaces. In Handbook of the geometry of Banach spaces, Vol. 2, pages 1459-1517. North-Holland, Amsterdam, 2003.

[10] Yves Raynaud. On ultrapowers of non commutative $L_{p}$ spaces. J. Operator Theory, 48(1):41-68, 2002.

[11] Éric Ricard. Hölder estimates for the noncommutative Mazur maps. Arch. Math. (Basel), 104(1):37-45, 2015.

[12] Éric Ricard. Fractional powers on noncommutative $L_{p}$ for $p<1$. Adv. Math., 333:194-211, 2018.

[13] Éric Ricard and Quanhua Xu. Complex interpolation of weighted noncommutative $L_{p}$-spaces. Houston J. Math., 37(4):1165-1179, 2011.

[14] Quanhua Xu. Applications du théorème de factorisation pour des fonctions à valeurs opérateurs. Studia Math., 95(3):273-292, 1990.

Normandie Univ, Unicaen, CNRS, Laboratoire de Mathématiques Nicolas Oresme, 14000 Caen, France

Email address: eric.ricard@unicaen.fr 